\title{
Basal cell carcinoma risk and solar UV exposure in occupationally relevant anatomic sites: do histological subtype, tumor localization and Fitzpatrick phototype play a role? A population-based case-control study
}

A. Bauer ${ }^{1 *+}$ D, E. Haufe ${ }^{2 \dagger}$, L. Heinrich ${ }^{2}$, A. Seidler ${ }^{3,4}$, H. J. Schulze ${ }^{5}$, P. Elsner ${ }^{6}$, H. Drexler ${ }^{7}$, S. Letzel ${ }^{8}$, S. M. John ${ }^{9}$, M. Fartasch ${ }^{10}$, T. Brüning ${ }^{10}$, S. Dugas-Breit ${ }^{5}$, M. Gina ${ }^{6}$, W. Weistenhöfer ${ }^{7}$, K. Bachmann ${ }^{11}$, I. Bruhn ${ }^{1}$, B. M. Lang ${ }^{12}$, R. Brans ${ }^{9}$, J. P. Allam ${ }^{13}$, W. Grobe ${ }^{13}$, S. Westerhausen ${ }^{14}$, P. Knuschke ${ }^{15}$, M. Wittlich ${ }^{14}$, T. L. Diepgen ${ }^{11}$, J. Schmitt ${ }^{2}$ and for the FB181 study group

\begin{abstract}
Background: A two-fold risk increase to develop basal cell carcinoma was seen in outdoor workers exposed to high solar UV radiation compared to controls. However, there is an ongoing discussion whether histopathological subtype, tumor localization and Fitzpatrick phototype may influence the risk estimates.

Objectives: To evaluate the influence of histological subtype, tumor localization and Fitzpatrick phototype on the risk to develop basal cell carcinoma in highly UV-exposed cases and controls compared to those with moderate or low solar UV exposure.

Methods: Six hundred forty-three participants suffering from incident basal cell carcinoma in commonly sunexposed anatomic sites (capillitium, face, lip, neck, dorsum of the hands, forearms outside, décolleté) of a population-based, case-control, multicenter study performed from 2013 to 2015 in Germany were matched to controls without skin cancer. Multivariate logistic regression analysis was conducted stratified for histological subtype, phototype 1/2 and 3/4. Dose-response curves adjusted for age, age ${ }^{2}$, sex, phototype and non-occupational UV exposure were calculated.

(Continued on next page)
\end{abstract}

\footnotetext{
* Correspondence: andrea.bauer@uniklinikum-dresden.de

${ }^{\dagger}$ A. Bauer and E. Haufe contributed equally to this work.

$\wedge$ Deceased.

'Department of Dermatology, University AllergyCentre, Medical Faculty Carl

Gustav Carus, Technical University Dresden, Fetscherstr 74, D-01307 Dresden, Germany

Full list of author information is available at the end of the article
}

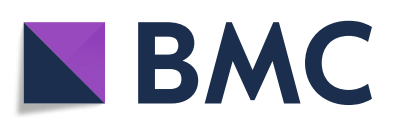

(- The Author(s). 2020 Open Access This article is licensed under a Creative Commons Attribution 4.0 International License, which permits use, sharing, adaptation, distribution and reproduction in any medium or format, as long as you give appropriate credit to the original author(s) and the source, provide a link to the Creative Commons licence, and indicate if changes were made. The images or other third party material in this article are included in the article's Creative Commons licence, unless indicated otherwise in a credit line to the material. If material is not included in the article's Creative Commons licence and your intended use is not permitted by statutory regulation or exceeds the permitted use, you will need to obtain permission directly from the copyright holder. To view a copy of this licence, visit http://creativecommons.org/licenses/by/4.0/. The Creative Commons Public Domain Dedication waiver (http://creativecommons.org/publicdomain/zero/1.0/) applies to the data made available in this article, unless otherwise stated in a credit line to the data. 
(Continued from previous page)

Results: Participants with high versus no (OR 2.08; 95\% Cl 1.24-3.50; $p=0.006)$ or versus moderate $(\mathrm{OR} 2.05 ; 95 \% \mathrm{Cl}$ 1.15-3.65; $p=0.015$ ) occupational UV exposure showed a more than two-fold significantly increased risk to develop BCC in commonly UV-exposed body sites. Multivariate regression analysis did not show an influence of phototype or histological subtype on risk estimates. The restriction of the analysis to BCC cases in commonly sun-exposed body sites did not influence the risk estimates. The occupational UV dosage leading to a 2-fold increased basal cell carcinoma risk was 6126 standard erythema doses.

Conclusion: The risk to develop basal cell carcinoma in highly occupationally UV-exposed skin was doubled consistently, independent of histological subtype, tumor localization and Fitzpatrick phototype.

Keywords: Occupation, Solar UV exposure, Basal cell carcinoma, Outdoor work, Histological subtype, Localization, Fitzpatrick phototype

\section{Background}

Outdoor workers are exposed to many different hazards like solar ultraviolet (UV) radiation and heat, pesticides and other chemicals as well as urban air pollutants and allergens that may lead to health problems [1-4]. This article focuses on solar UV radiation.

Exposure to solar UV-radiation is the most important risk factor for the development of cutaneous basal cell carcinoma (BCC) [5]. Outdoor workers are exposed to significantly higher occupational UV exposure dosages than the rest of the population [6-8]. A recent metaanalysis of the epidemiological literature [9] $(n=23$ studies) revealed a $43 \%$ increase in BCC risk in occupationally UV-exposed workers compared to indoor workers (OR 1.43; 95\% CI 1.23-1.66; $p=0.0001$ ). However, based on the available epidemiological evidence at this time, informed decision making to consider BCC related to occupational UV exposure as an occupational disease was not possible. This was due to the limited methodological quality of almost all included studies. Quality was low because of low numbers of included participants, poorly classified indoor and outdoor tasks and occupations, lack of quantification of occupational and leisure time UV exposure, lack of controlling for relevant confounders and high risk of bias [9]. A recent population-based case-control study [10] that included consecutive patients with incident BCC $(n=836)$ overcame limitations of former studies. Previous work from our group showed that individuals with high occupational solar UV exposure had a 1.84-fold significantly increased BCC risk (95\% CI 1.19-2.83) compared with no occupational UV exposure and a 1.97-fold significantly increased BCC risk (95\% CI 1.20-3.22) compared with moderate occupational UV exposure [10].

However, the study population of Schmitt et al. [10] consisted of a population-based sample of $\mathrm{BCC}$ patients with no special focus on occupational skin cancer. BCC were localized on all parts of the body. To analyze whether the occurrence of BCC is dependent on high occupational solar UV exposure in outdoor workers, further analyses were requested by the experts of the research monitoring group of FB-181 for commonly sunexposed, occupationally relevant body sites $(n=643$ out of 836 BCC in total), different histopathological subtypes and Fitzpatrick phototype [11, 12].

The aim of this study was to determine the extent to which the established significant association of occupational solar UV exposure and the occurrence of BCC varies depending on histological subtype, tumor localization and Fitzpatrick phototype. The analyses took the influence of age, age ${ }^{2}$, sex and non-occupational UV exposure into account.

The primary study questions were:

1. The risk to develop BCC at capillitium, face, lip, neck, dorsum of the hands, forearms outside or décolleté regarding high ( $\geq 90$ th percentile) versus moderate (44th-60th percentile) occupational UV exposure is influenced by histopathologic BCC subtype (nodular, sclerodermiform, superficial).

2. The risk to develop BCC at capillitium, face, lip, neck, dorsum of the hands, forearms outside or décolleté is influenced by tumor localization and Fitzpatrick phototype.

3. There is a positive dose-response relationship between cumulative solar UV exposure and the risk of developing BCC at capillitium, face, lip, neck, dorsum of the hands, forearms outside or décolleté.

\section{Methods}

Study design, setting, eligibility criteria for cases and controls

Six hundred forty-three cases suffering from incident BCC (first diagnosis of histologically confirmed BCC within the past 2 years, age $\geq 30$ years) in commonly sun-exposed body sites (capillitium, face, lip, neck, dorsum of the hands, forearms outside, décolleté) out of 836 overall cases of a population-based, case-control, multicenter study performed from 2013 to 2015 in 8 German study centres [10] were included in the current 
post-hoc analysis. Cases with incident BCC in other body sites $(n=193)$, where occupational UV exposure is less likely, were excluded (upper arm, abdomen, back, buttocks, lower extremities). Sex and age matched controls (age $\geq 30$ years) without a history of skin cancer were recruited from regional residents' registration offices by mail. They were offered physical examination and expense allowance for study participation. In case of non-response, two reminders were sent out by mail. Individuals who responded (response rate 33.9\%) to participate as potential controls received a standardized interview and full body dermatological examination by a trained dermatologist at the local recruitment site. Patients suffering from Xeroderma pigmentosum, GorlinGoltz-Syndrome or diseases with a genetic background that may result in $\mathrm{BCC}$ development were excluded.

\section{Interviews, clinical examinations, assessment of occupational and non-occupational UV exposure}

Trained investigators (Residents and Consultant Dermatologists) performed standardized interviews, full body clinical examinations, including photo-damage scoring of 12 different body sites (capillitium, face, neck, back of the hand, upper arm inside, forearm and upper arm outside, décolleté, abdomen, back, buttocks, lower extremities), computer-assisted UV exposure assessments and non-occupational UV exposure assessments. Trained experts of the German Social Accident Insurance Institutions performed occupational UV-exposure assessments. Occupational and non-occupational UV exposure was assessed in detail. To reduce recall bias the participants were asked in the invitation letter to consider the major stages of their private life (e.g. childhood/school years, period of training, entry into working life, partnerships, family, possibly retirement from working life) as well as the duration of each period, place of residence, typical behavior in leisure time and on holidays, prior to the interview at the clinic. UV protection behavior was assessed in detail for leisure time. To detect occupational UV exposure and protection behavior, participants were asked about the major stages of their professional lives as well as the duration of and the activities in each period. Furthermore, they were asked at what times of the day and how long they usually spent time outdoors during their working life. Working periods abroad, e.g. on assembly work, and exposure to artificial UV radiation (e.g. during welding) should be remembered, too. Moreover, occupational UV exposure was assessed more precisely compared to previous studies by using insurance documents on the duration of outdoor occupations for the calculation of the cumulative occupational UV exposure. A detailed description of the calculation of the total non-occupational and occupational UV exposure was recently published elsewhere [10]. The investigators assessing occupational UV exposure were blinded concerning case or control status of the study participants. Leisure time UV exposure was validated by a blinded investigator using previously specified algorithms.

\section{Publication of study protocol}

The study protocol was published in the project registry of the German Federal Ministry of Labour and Social Affairs (http://www.bmas.de/DE/Themen/Arbeitsschutz/ Forschungsdatenbank/UVT/DGUV-FB_181_Hautkrebs_ durch_UV-Strahlung_IFA4206.html) prior to the start of the study.

\section{Statistical analysis}

Descriptive analyses were performed considering variable types and distributions. Conditional logistic regression analysis was used to estimate odds ratios (OR) and their 95\% confidence intervals (CI) for BCC with total, occupational and non-occupational lifetime UV exposure. For the stratified analyses by phototype it was necessary to break the propensity-score matched pairs. All cases with $\mathrm{BCC}$ in sun-exposed body sites and with Fitzpatrick phototype $1 / 2$ or $3 / 4$ were compared to all controls with the respective Fitzpatrick phototype using logistic regression. Regression analyses were adjusted for sex, age, age ${ }^{2}$ and Fitzpatrick phototype. Regression analyses for occupational UV exposure were additionally adjusted for non-occupational UV exposure.

To investigate the dose-response relationship between lifetime total, occupational and non-occupational UV exposure (in SED) in commonly sun-exposed body sites and $\mathrm{BCC}$ risk, we first assessed empirical dose-risk relationships by calculating the OR and corresponding 95\% CI for each exposure dosage by conditional logistic regression. Assuming a non-linear dose-response relationship [13], the empirical dose-response functions were fitted to the observed data using fractional polynomials in the linear regression models [14] aiming to maximize the $\mathrm{R}$ squared. Sample size calculation was performed with GPower 3.1 (Statistical Power Analyses for Windows, University of Düsseldorf). Assuming a statistical power of $80 \%$ and a probability of error of $5 \%$, a total of at least 515 cases with BCC in commonly UV-exposed body sites must be included in the analyses for detecting a doubling of BCC risk for highly UV-exposed persons. SPSS 25 (IBM, Armonk, NY) and Stata 14 (Stata Corp, College Station, TX) were used for data analysis.

\section{Results}

\section{Characteristics of the study population}

Six hundred forty-three (81.6\%) participants with incident BCC in commonly sun-exposed body sites (capillitium, face, lip, neck, dorsum of the hands, forearms outside, décolleté) out of 836 cases with incident BCC 
from the entire study population of Schmitt et al. [10], as well as matched controls were included in the analysis. Among cases, $40.4 \%$ had left school after 9 years compared to $27.8 \%$ among controls (Fisher's exact test for education of cases versus controls: $p<0.001)$. Total occupational UV exposure was considerably higher in cases than controls (cases 1934.4 SED; controls 1317.6 SED; Wilcoxon rank-sum test: $p=0.0642$ ). The difference in lifetime UV exposure was mainly attributed to differences in occupational exposure. No gender difference was seen, indicating successful matching (Table 1).

\section{Histopathological subtype and tumor localization}

Table 2 shows the distribution of histopathological subtypes of BCC in 643 male and female participants with BCC in commonly sun-exposed body sites. Nodular BCC $(n=403,62.7 \%)$ was the most common histopathological subtype followed by sclerodermiform $(n=145$, $22.6 \%)$ and superficial $(n=28,4.4 \%)$ BCC (Table 2). No significant difference in distribution of these three BCC subtypes was seen between females and males $(n=576$, $p=0.087)$.

The mean age of tumor manifestation was slightly higher in superficial (68.3 \pm 8.1 years of age) compared to nodular (66.1 \pm 10.8 years of age) and sclerodermiform $(66.0 \pm 11.4$ years of age) BCC.

Table 3 shows the distribution of histopathologic subtypes on distinct anatomical localizations in commonly sun-exposed body sites. Anatomical localizations differed significantly between the BCC subtypes (Pearson's chisquared test $p<0.001$ ). Nodular and sclerodermiform BCC were mainly localized on the head (face, capillitium and lips). Superficial BCC predominated in the décolleté (Table 3).

\section{Estimation of BCC risk}

Participants with a high total UV exposure showed a 2fold significantly increased risk to develop $\mathrm{BCC}$ in sunexposed body sites versus low total UV exposure (OR 2.24 ; $95 \%$ CI $1.20-4.19 ; p=0.012$ ). The analyses for occupational UV exposure yielded significantly increased $\mathrm{BCC}$ risks for high versus no occupational UV exposure (OR 2.08; 95\% CI 1.24-3.50; $p=0.006$ ) and high versus moderate occupational UV exposure (OR 2.05; 95\% CI $1.15-3.65 ; p=0.015$ ) (Table 4).

\section{Estimation of $B C C$ risk for different histopathological $B C C$ subtypes}

Participants with a high ( $\geq$ 90th percentile) total UV exposure showed a more than 2-fold significantly increased risk to develop nodular, sclerodermiform or superficial BCC in sun-exposed body sites (OR 2.27; 95\% CI 1.174.42; $p=0.016)$ compared to participants with low total UV exposure. Similar results were observed for high versus low occupational UV exposure (OR 1.98; 95\% CI $1.14-3.44 ; p=0.015)$ and high versus moderate occupational UV exposure (OR 2.02; 95\% CI 1.09-3.74; $p=$ 0.026) (Table 5). There were no differences in the increased risk considering the histological subtypes. However, the number of cases was not sufficient to generate significant risk estimates (Tables S1-S3 supplementary material).

\section{Estimation of BCC risk for different Fitzpatrick phototypes}

Among other potential confounders (age, age ${ }^{2}$, sex), phototype was included in the risk estimation of BCC. For total UV exposure, the OR of phototype was 0.76 (95\% CI 0.61-0.94; $p=0.012$ ); for occupational UV exposure it was 0.79 (95\% CI $0.64-0.98, p=0.034)$. The stratified analyses of BCC risk (BCC in occupationally sun-exposed body sites, $n=643$ ) by phototype showed no relevant differences of the risk estimates in participants with Fitzpatrick phototype $1 / 2\left(\mathrm{n}_{\text {cases }}=411, \mathrm{n}_{\text {con- }}\right.$ trols $=491)$ and phototype $3 / 4\left(\mathrm{n}_{\text {cases }}=232, \mathrm{n}_{\text {controls }}=\right.$ 342 ). The risk to develop BCC in commonly sunexposed body sites was doubled for high versus no occupational UV exposure in phototype 1/2 (OR 1.73; 95\% CI $1.05-2.85 ; p=0.031$ ) as well as phototype $3 / 4$ (OR 2.13; 95\% CI 1.12-4.05; $p=0.021$ ). Similar results were obtained by comparing $\mathrm{BCC}$ risk for high versus moderate occupational UV exposure in phototype 1/2 (OR 2.11; $95 \%$ CI $1.21-3.68 ; p=0.009)$ and phototype $3 / 4$ (OR 1.88; 95\% CI 0.94-3.75; $p=0.072$; not statistically significant) (Tables 6 and 7).

\section{Occupations under risk}

Outdoor occupations under risk in the highly occupationally UV-exposed group ( $\geq 5870.5$ SED) included agriculture, animal and plant production, building and construction work, outdoor metal work, road construction and civil engineering, occupations in transport, street and vehicle cleaning as well as security staff and engineers with preponderant outdoor tasks.

\section{Dose-response relationship between UV exposure and $B C C$ risk}

Adjusted dose-response curves for cases with BCC in commonly sun-exposed body sites and propensity-score matched controls ( $n=643$ in each group) were estimated for total, occupational and non-occupational UV exposure (Figs. 1, 2 and 3). Positive dose-response curves were observed especially for occupational UV exposure, with a doubling dose of 6126 SED for the risk to develop BCC in commonly sun-exposed body sites (Fig. 2).

\section{Discussion}

In the present study, we demonstrated that BCC risk estimates in persons with high occupational UV exposure 
Table 1 Characteristics of study population

\begin{tabular}{|c|c|c|c|}
\hline & \multicolumn{2}{|l|}{ study population } & \multirow[t]{2}{*}{$P$ values } \\
\hline & $\begin{array}{l}\text { cases with BCC in occupationally UV-exposed } \\
\text { body sites }(n=643)\end{array}$ & controls $(n=643)$ & \\
\hline \multicolumn{4}{|l|}{ Sociodemographic characteristics } \\
\hline Age in years (mean $\pm S D$, range) & $66.3 \pm 10.9(29-89)$ & $66.2 \pm 9.7(31-83)$ & $\begin{array}{l}\text { not applicable (Propensity } \\
\text { score matched) }\end{array}$ \\
\hline \multicolumn{4}{|l|}{$\operatorname{Sex}(n, \%)$} \\
\hline Male & $364(56.6 \%)$ & $368(57.2 \%)$ & \multirow{2}{*}{$\begin{array}{l}\text { not applicable (Propensity } \\
\text { score matched) }\end{array}$} \\
\hline Female & $279(43.4 \%)$ & $275(42.8 \%)$ & \\
\hline \multicolumn{4}{|l|}{ Education (n, \%) } \\
\hline No graduation & $3(0.5 \%)$ & $1(0.2 \%)$ & \multirow[t]{5}{*}{ Fisher's exact test: $p<0.001$} \\
\hline 9 years & $260(40.4 \%)$ & $179(27.8 \%)$ & \\
\hline Middle school (10 years) & $153(23.8 \%)$ & $141(21.9 \%)$ & \\
\hline High school (12 years) & $172(26.7 \%)$ & $254(39.5 \%)$ & \\
\hline other & $55(8.6 \%)$ & $68(10.6 \%)$ & \\
\hline \multicolumn{4}{|l|}{ Clinical and anamnestic parameters } \\
\hline \multicolumn{4}{|l|}{ Fitzpatrick phototype (n, \%) } \\
\hline 1 & $46(7.2 \%)$ & $31(4.8 \%)$ & \multirow{5}{*}{$\begin{array}{l}\text { Pearson's chi-squared test: } \\
p=0.014\end{array}$} \\
\hline 2 & $365(56.8 \%)$ & $338(52.6 \%)$ & \\
\hline 3 & $223(34.7 \%)$ & $253(39.3 \%)$ & \\
\hline 4 & $9(1.4 \%)$ & 19 (2.95\%) & \\
\hline 5 & $0(0.00 \%)$ & $2(0.31 \%)$ & \\
\hline \multicolumn{4}{|c|}{ Immuno-suppressant agents (intake), current and/or previous (n, \%) } \\
\hline yes & $55(8.6 \%)$ & $38(5.9 \%)$ & \multirow{2}{*}{$\begin{array}{l}\text { Pearson's chi-squared test: } \\
p=0.067\end{array}$} \\
\hline no & $588(91.4 \%)$ & $605(94.1 \%)$ & \\
\hline \multicolumn{4}{|c|}{ Positive family history for skin cancer (n, \%) } \\
\hline yes & $89(13.8 \%)$ & $61(9.5 \%)$ & \multirow{2}{*}{$\begin{array}{l}\text { Pearson's chi-squared test: } \\
p=0.015\end{array}$} \\
\hline no & $554(86.2 \%)$ & $582(90.5 \%)$ & \\
\hline \multicolumn{4}{|l|}{ Migration background (n, \%) } \\
\hline yes & $35(5.4 \%)$ & $31(4.8 \%)$ & \multirow{2}{*}{$\begin{array}{l}\text { Pearson's chi-squared test: } \\
p=0.613\end{array}$} \\
\hline no & $608(94.6 \%)$ & $612(95.2 \%)$ & \\
\hline \multicolumn{4}{|c|}{ UV exposure Standard Erythema Dose (SED; mean \pm SD, range), } \\
\hline Total UV exposure & $12,520.6 \pm 4473.1(4601.2-38,409.2)$ & $12,002.2 \pm 3976.1(3980.9-30,984.9)$ & $\begin{array}{l}\text { Wilcoxon rank-sum test: } \\
p=0.0642\end{array}$ \\
\hline Occupational UV exposure (total) & $1934.4 \pm 3360.7(0.0-22,763.6)$ & $1317.6 \pm 2439.1(0.0-14,984.0)$ & $\begin{array}{l}\text { Wilcoxon rank-sum test: } \\
p=0.0182\end{array}$ \\
\hline $\begin{array}{l}\text { Persons with occupational UV } \\
\text { exposure }>0 \text { SED }\end{array}$ & $375(58.3 \%)$ & $357(55.5 \%)$ & $\begin{array}{l}\text { Pearson's chi-squared test: } \\
p=0.311\end{array}$ \\
\hline $\begin{array}{l}\text { Occupational UV exposure for } \\
\text { exposed ( }>0 \text { SED) persons }\end{array}$ & $3316.9 \pm 3845.8(14.4-22,763.6)$ & $2373.2 \pm 2866.5(3.8-14,984.0)$ & $\begin{array}{l}\text { Wilcoxon rank-sum test: } \\
p=0.0011\end{array}$ \\
\hline $\begin{array}{l}\text { Non-occupational UV exposure } \\
\text { (total) }\end{array}$ & $10,586.2 \pm 2708.2(4460.3-20,988.9)$ & $10,684.6 \pm 2878.0(3980.9-30,984.9)$ & $\begin{array}{l}\text { Wilcoxon rank-sum test: } \\
p=0.7421\end{array}$ \\
\hline $\begin{array}{l}\text { Non-occupational UV exposure } \\
\text { (vacation) }\end{array}$ & $5541.0 \pm 2025.5(1566.7-16,122.0)$ & $5654.3 \pm 2170.1(1663.6-14,992.0)$ & $\begin{array}{l}\text { Wilcoxon rank-sum test: } \\
p=0.6347\end{array}$ \\
\hline $\begin{array}{l}\text { Non-occupational UV exposure } \\
\text { (leisure time) }\end{array}$ & $5052.2 \pm 1373.8(1624.7-13,338.0)$ & $5032.2 \pm 1387.8(1235.1-17,459.9)$ & $\begin{array}{l}\text { Wilcoxon rank-sum test: } \\
p=0.5493\end{array}$ \\
\hline
\end{tabular}


Table 2 Distribution of all histopathologic BCC subtypes in males and females $(n=643)$

\begin{tabular}{llll}
\hline Histopathologic subtype $^{\mathbf{a}}$ & $\mathrm{n}(\%)$ & Male & Female \\
\hline Nodular & $403(62.7 \%)$ & $230(63.2 \%)$ & $173(62.0 \%)$ \\
Sclerodermiform & $145(22.6 \%)$ & $69(19.0 \%)$ & $76(27.2 \%)$ \\
Superficial & $28(4.4 \%)$ & $18(4.9 \%)$ & $10(3.6 \%)$ \\
Other (ulcus rodens, ulcus terebrans, pigmented) & $31(4.8 \%)$ & $21(5.8 \%)$ & $10(3.6 \%)$ \\
No data on subtype available & $36(5.6 \%)$ & $26(7.1 \%)$ & $10(3.6 \%)$ \\
Total & 643 & 364 & 279 \\
\hline
\end{tabular}

$n$ number, \% percentage

a in commonly sun-exposed body sites

in commonly sun-exposed body sites were doubled consistently, irrespective of histological subtype, tumor localization and Fitzpatrick phototype. This analysis allowed us to substantiate the results of the recently published population-based, case-control study in patients with incident BCC [10]. The previous analysis yielded a doubled risk to develop BCC in persons with high compared to low or moderate occupational solar UV exposure but included BCC not localized in commonly sun-exposed body sites [10].

\section{Association of histopathological BCC subtype and risk estimates}

In our data set the majority of nodular and sclerodermiform BCC were found on chronically sun-exposed body areas like head and neck. Superficial BCC were more often diagnosed on the trunk, where exposition to high intermittent UV exposures is more likely. In the literature comparable distribution patterns of histological subtypes are published [15-21]. Different susceptibilities to different sun exposure patterns and amounts have been discussed as possible reasons for the different distribution of histological subtypes on head and neck compared to trunk [12, 15-21].

To evaluate whether the different histological subtypes are differently related to occupational and nonoccupational UV exposure, we performed multivariate regression analyses, adjusted for age, age ${ }^{2}$, sex and phototype. We found a consistently doubled risk to develop BCC in commonly sun-exposed body sites (capillitium, face, lip, neck, dorsum of the hands, forearms, décolleté) in participants with high versus low and moderate occupational UV exposure, respectively. We did not see any differences in the risk estimates in different histological subtypes for high cumulative UV exposure. Pelucchi et al. [12] showed a significantly increased risk for nodular (OR 1.53; 95\% CI 1.08-2.18) but not for superficial (OR 0.71; 95\%CI 0.44-1.15) BCC in patients exposed to occupational solar UV radiation. However, like in our study, subgroup analysis in cases with long duration occupational UV exposure revealed no significant risk increase for nodular BCC [12]. This indicates, if a certain threshold of occupational UV exposure is exceeded, the histological BCC subtype is irrelevant.

\section{Association of Fitzpatrick phototype and BCC risk}

Concerning the association of Fitzpatrick phototype and BCC risk our study indicates that individuals with phototype $3 / 4$ are at lower risk compared to individuals with phototype $1 / 2$ for UV exposure in general as well as for occupational UV exposure. Importantly, high occupational UV-exposure was as harmful for individuals with phototype $1 / 2$ as for those with phototype $3 / 4$ leading to an approximately 2 -fold increased risk for BCC on

Table 3 Anatomical distribution of BCC subtypes in commonly sun-exposed body sites $(n=643)^{*}$

\begin{tabular}{|c|c|c|c|c|c|c|}
\hline Histopathologic subtype & Nodular & Sclerodermiform & Superficial & Other & No data available & All \\
\hline Capillitium & $29(7.2 \%)$ & $15(10.3 \%)$ & 0 & $2(6.5 \%)$ & $4(11.1 \%)$ & $50(7.8 \%)$ \\
\hline Face & $335(83.1 \%)$ & $110(75.9 \%)$ & $9(32.1 \%)$ & $23(74.2 \%)$ & $19(52.8 \%)$ & $496(77.1 \%)$ \\
\hline Lip & $3(0.7 \%)$ & $4(2.8 \%)$ & $1(3.6 \%)$ & $1(3.2 \%)$ & $1(2.8 \%)$ & $10(1.6 \%)$ \\
\hline Neck & $12(3.0 \%)$ & $5(3.5 \%)$ & $1(3.6 \%)$ & $1(3.2 \%)$ & $2(5.6 \%)$ & $21(3.3 \%)$ \\
\hline Back of hand & $2(0.5 \%)$ & 0 & $1(3.6 \%)$ & 0 & $1(2.8 \%)$ & $4(0.6 \%)$ \\
\hline Forearm outside & $3(0.7 \%)$ & $2(1.4 \%)$ & 0 & 0 & $3(8.3 \%)$ & $8(1.2 \%)$ \\
\hline Décolleté & $19(4.7 \%)$ & $9(6.2 \%)$ & $16(57.1 \%)$ & $4(12.9 \%)$ & $6(16.7 \%)$ & $54(8.4 \%)$ \\
\hline Total & $403(100 \%)$ & 145 (100\%) & $28(100 \%)$ & $31(100 \%)$ & $36(100 \%)$ & $643(100 \%)$ \\
\hline
\end{tabular}

* Pearson's chi-squared test showed a significant association between body sites classified into head (capillitium/face/lip) versus the remaining body sites and BCC subtypes (nodular, sclerodermiform and superficial), $X^{2}(2)=75.3651, p<0.001$ 
Table 4 Association of total and occupational UV exposure and risk for BCC in commonly sun-exposed body sites ( $n=643$ in each group)

\begin{tabular}{|c|c|c|c|c|c|c|c|c|}
\hline UV exposure & Cases $^{\mathbf{a}}$ & Contr. $^{a}$ & $O R^{\mathbf{b}}$ & $(95 \%-C l)^{\mathbf{b}}$ & $\mathrm{p}$ & $\mathrm{OR}^{c}$ & $(95 \%-C I)^{c}$ & $p$ \\
\hline \multicolumn{9}{|c|}{ Total UV exposure (leisure time and occupational exposure) } \\
\hline$<$ 20th percentile $(<8985.0$ SED) & 115 & 143 & Ref. & Ref. & Ref. & . & & \\
\hline 20. $-<40$ th percentile $(8985.0-10,632.6$ SED) & 134 & 131 & 1.80 & $1.05-3.10$ & 0.034 & & . & \\
\hline 40th $-<60$ th percentile $(10,632.7-12,565.6$ SED $)$ & 138 & 132 & 1.70 & $1.01-2.84$ & 0.044 & . & . & . \\
\hline 60th $-<90$ th percentile $(12,565.7-18,006.7$ SED $)$ & 190 & 183 & 1.73 & $1.05-2.86$ & 0.033 & . & & . \\
\hline$\geq 90$ th percentile ( $\geq 18,006.8$ SED) & 66 & 54 & 2.24 & $1.20-4.19$ & 0.012 & . & . & $\cdot$ \\
\hline \multicolumn{9}{|l|}{ Total UV exposure (high versus moderate exposure) } \\
\hline 40th $-<60$ th percentile $(10,632.7-12,565.6$ SED) & 138 & 132 & Ref. & Ref. & Ref. & & . & \\
\hline$\geq 90$ th percentile ( $\geq 18,006.8$ SED) & 66 & 54 & 1.32 & $0.81-2.17$ & 0.269 & . & . & \\
\hline \multicolumn{9}{|l|}{ Occupational UV exposure } \\
\hline$<44$ th percentile $(<2.9$ SED) & 268 & 286 & Ref. & Ref. & Ref. & Ref. & Ref. & Ref. \\
\hline 44th $-<60$ th percentile (2.9-532.1 SED) & 94 & 115 & 1.02 & $0.68-1.53$ & 0.914 & 1.02 & $0.68-1.52$ & 0.935 \\
\hline 60th $-<90$ th percentile (532.2-5870.4 SED) & 206 & 201 & 1.13 & $0.81-1.56$ & 0.474 & 1.13 & $0.82-1.56$ & 0.459 \\
\hline$\geq$ 90th percentile ( $\geq 5870.5$ SED) & 75 & 41 & 2.08 & $1.24-3.48$ & 0.006 & 2.08 & $1.24-3.50$ & 0.006 \\
\hline \multicolumn{9}{|c|}{ Occupational UV exposure (high versus moderate exposure) } \\
\hline 44th $-<60$ th percentile (2.9-532.1 SED) & 94 & 115 & Ref. & Ref. & Ref. & Ref. & Ref. & Ref. \\
\hline$\geq 90$ th percentile ( $\geq 5870.5$ SED) & 75 & 41 & 2.03 & $1.14-3.61$ & 0.016 & 2.05 & $1.15-3.65$ & 0.015 \\
\hline
\end{tabular}

a number of cases and controls (contr)

b adjusted for age, age ${ }^{2}$, sex, phototype

c adjusted for age, age ${ }^{2}$, sex, phototype and non-occupational UV exposure

Table 5 Association of UV exposure and risk for BCC in cases with histopathological subtypes nodular, sclerodermiform or superficial and matched controls ( $n=576$ in each group)

\begin{tabular}{lllllllll}
\hline UV exposure & Cases $^{\mathbf{a}}$ & Contr. $^{\mathbf{a}}$ & $\mathrm{OR}^{\mathbf{b}}$ & $(95 \%-\mathrm{Cl})^{\mathbf{b}}$ & $\mathrm{p}$ & $\mathrm{OR}^{\mathbf{c}}$ & $\left(95 \%-\mathrm{Cl}^{\mathbf{c}}\right.$ & $\mathrm{p}$
\end{tabular}

Total UV exposure (leisure time and occupational exposure)

$\begin{array}{llllll}<\text { 20th percentile }(<8985.0 \text { SED }) & 107 & 132 & \text { Ref. } & \text { Ref. } & \text { Ref. } \\ \text { 20. }-<\text { 40th percentile }(8985.0-10,632.6 \text { SED }) & 117 & 119 & 1.74 & 0.98-3.10 & 0.058 \\ \text { 40th }-<60 \text { th percentile }(10,632.7-12,565.6 \text { SED) } & 125 & 118 & 1.71 & 0.99-2.94 & 0.053 \\ \text { 60th }-<90 \text { th percentile }(12,565.7-18,006.7 \text { SED }) & 170 & 160 & 1.87 & 1.10-3.18 & 0.021 \\ \geq 90 \text { th percentile }(\geq 18,006.8 \text { SED) } & 57 & 47 & 2.27 & 1.17-4.42 & 0.016\end{array}$

Total UV exposure (high versus moderate exposure)

$\begin{array}{llllll}\text { 40th }-<60 \text { th percentile }(10,632.7-12,565.6 \text { SED }) & 125 & 118 & \text { Ref. } & \text { Ref. } & \text { Ref. } \\ \geq \text { 90th percentile }(\geq 18,006.8 \text { SED) } & 57 & 47 & 1.33 & 0.78-2.26 & 0.292\end{array}$

\section{Occupational UV exposure}

\begin{tabular}{|c|c|c|c|c|c|c|c|c|}
\hline$<44$ th percentile $(<2.9$ SED) & 251 & 264 & Ref. & Ref. & Ref. & Ref. & Ref. & Ref. \\
\hline 44th $-<60$ th percentile (2.9-532.1 SED) & 78 & 101 & 0.98 & $0.64-1.51$ & 0.933 & 0.98 & $0.63-1.52$ & 0.931 \\
\hline 60th $-<90$ th percentile (532.2-5870.4 SED) & 183 & 173 & 1.13 & $0.80-1.58$ & 0.49 & 1.13 & $0.80-1.58$ & 0.49 \\
\hline$\geq$ 90th percentile ( $\geq 5870.5$ SED) & 64 & 38 & 1.98 & $1.14-3.44$ & 0.015 & 1.98 & $1.14-3.44$ & 0.015 \\
\hline \multicolumn{9}{|c|}{ Cccupational UV exposure (high versus moderate exposure) } \\
\hline 44th $-<60$ th percentile (2.9-532.1 SED) & 78 & 101 & Ref. & Ref. & Ref. & Ref. & Ref. & Ref. \\
\hline$\geq 90$ th percentile ( $\geq 5870.5$ SED) & 64 & 38 & 2.02 & $1.09-3.74$ & 0.026 & 2.02 & $1.09-3.75$ & 0.026 \\
\hline
\end{tabular}

a number of cases and controls (contr)

b adjusted for age, age ${ }^{2}$, sex, phototype

c adjusted for age, age ${ }^{2}$, sex, phototype and non-occupational UV exposure 
Table 6 Association of total and occupational UV exposure and risk for BCC in phototypes $1 / 2$ and matched controls ( $n=451$ in each group)

\begin{tabular}{|c|c|c|c|c|c|c|c|c|}
\hline UV exposure & Cases $^{\mathbf{a}}$ & Contr. $^{\text {a }}$ & $\mathrm{OR}^{\mathbf{b}}$ & $(95 \%-C l)^{\mathbf{b}}$ & $\mathrm{P}$ & $\mathrm{OR}^{\mathrm{c}}$ & $(95 \%-\mathrm{Cl})^{\mathbf{c}}$ & $\mathrm{p}$ \\
\hline \multicolumn{9}{|c|}{ Total UV exposure (leisure time and occupational exposure) } \\
\hline$<$ 20th percentile $(<8985.0$ SED $)$ & 75 & 120 & Ref. & Ref. & Ref. & . & . & . \\
\hline 20. $-<$ 40th percentile $(8985.0-10,632.6$ SED) & 90 & 102 & 1.71 & $1.10-2.66$ & 0.018 & . & . & 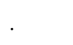 \\
\hline 40th - <60th percentile $(10,632.7-12,565.6$ SED $)$ & 95 & 95 & 1.92 & $1.21-3.03$ & 0.005 & . & & $\cdot$ \\
\hline 60th - <90th percentile $(12,565.7-18,006.7$ SED $)$ & 111 & 136 & 1.60 & $1.02-2.51$ & 0.042 & & . & \\
\hline$\geq$ 90th percentile ( $\geq 18,006.8$ SED) & 40 & 38 & 2.12 & $1.17-3.85$ & 0.013 & $\cdot$ & . & \\
\hline \multicolumn{9}{|l|}{ Total UV exposure (high versus moderate exposure) } \\
\hline 40th - <60th percentile $(10,632.7-12,565.6$ SED $)$ & 95 & 95 & Ref. & Ref. & Ref. & & . & \\
\hline$\geq$ 90th percentile ( $\geq 18,006.8$ SED) & 40 & 38 & 1.11 & $0.64-1.90$ & 0.714 & . & & \\
\hline \multicolumn{9}{|l|}{ Occupational UV exposure } \\
\hline$<44$ th percentile $(<2.9$ SED) & 180 & 217 & Ref. & Ref. & Ref. & Ref. & Ref. & Ref. \\
\hline 44th - <60th percentile (2.9-532.1 SED) & 54 & 80 & 0.82 & $0.55-1.24$ & 0.353 & 0.82 & $0.55-1.24$ & 0.353 \\
\hline 60th - <90th percentile (532.2-5.870.4 SED) & 128 & 158 & 0.95 & $0.68-1.32$ & 0.759 & 0.95 & $0.68-1.32$ & 0.755 \\
\hline$\geq$ 90th percentile ( $\geq 5870.5$ SED) & 49 & 36 & 1.73 & $1.05-2.85$ & 0.031 & 1.73 & $1.05-2.85$ & 0.031 \\
\hline \multicolumn{9}{|c|}{ Occupational UV exposure (high versus moderate exposure) } \\
\hline 44th - <60th percentile (2.9-532.1 SED) & 54 & 80 & Ref. & Ref. & Ref. & Ref. & Ref. & Ref. \\
\hline$\geq$ 90th percentile ( $\geq 5870.5$ SED) & 49 & 36 & 2.11 & $1.21-3.68$ & 0.009 & 2.11 & $1.21-3.68$ & 0.009 \\
\hline
\end{tabular}

a number of cases and controls (contr)

b adjusted for age, age ${ }^{2}$, sex, phototype

c adjusted for age, age ${ }^{2}$, sex, phototype and non-occupational UV exposure

Table 7 Association of total and occupational UV exposure and risk for BCC in commonly sun-exposed body sites in phototypes 3/4 and matched controls ( $n=287$ in each group)

\begin{tabular}{|c|c|c|c|c|c|c|c|c|}
\hline UV exposure & Cases $^{\mathbf{a}}$ & Contr. $^{a}$ & $\mathrm{OR}^{\mathbf{b}}$ & $(95 \%-C l)^{\mathbf{b}}$ & $p$ & $\mathrm{OR}^{c}$ & $(95 \%-\mathrm{Cl})^{\mathbf{c}}$ & $\mathrm{p}$ \\
\hline \multicolumn{9}{|c|}{ Total UV exposure (leisure time and occupational exposure) } \\
\hline$<$ 20th percentile $(<8985.0$ SED) & 40 & 55 & Ref. & Ref. & Ref. & . & & \\
\hline 20. - < 40th percentile (8985.0 - 10,632.6 SED) & 44 & 66 & 1.11 & $0.61-2.04$ & 0.724 & . & . & . \\
\hline 40th $-<60$ th percentile $(10,632.7-12,565.6$ SED $)$ & 43 & 70 & 1.08 & $0.58-2.00$ & 0.805 & . & & \\
\hline 60th $-<90$ th percentile $(12,565.7-18,006.7$ SED $)$ & 79 & 117 & 1.25 & $0.69-2.24$ & 0.459 & . & . & . \\
\hline$\geq 90$ th percentile ( $\geq 18,006.8$ SED) & 26 & 34 & 1.58 & $0.75-3.33$ & 0.228 & . & . & \\
\hline \multicolumn{9}{|l|}{ Total UV exposure (high versus moderate exposure) } \\
\hline 40th $-<60$ th percentile $(10,632.7-12,565.6$ SED $)$ & 43 & 70 & Ref. & Ref. & Ref. & . & . & . \\
\hline$\geq 90$ th percentile ( $\geq 18,006.8$ SED) & 26 & 34 & 1.46 & $0.76-2.81$ & 0.254 & & . & . \\
\hline \multicolumn{9}{|l|}{ Occupational UV exposure } \\
\hline$<44$ th percentile $(<2.9$ SED) & 88 & 146 & Ref. & Ref. & Ref. & Ref. & Ref. & Ref. \\
\hline 44th $-<60$ th percentile (2.9-532.1 SED) & 40 & 63 & 1.14 & $0.69-1.86$ & 0.61 & 1.13 & $0.69-1.86$ & 0.618 \\
\hline 60th $-<90$ th percentile (532.2-5.870.4 SED) & 78 & 107 & 1.39 & $0.92-2.10$ & 0.123 & 1.39 & $0.92-2.10$ & 0.122 \\
\hline$\geq 90$ th percentile ( $\geq 5870.5$ SED) & 26 & 26 & 2.13 & $1.12-4.05$ & 0.021 & 2.13 & $1.12-4.06$ & 0.021 \\
\hline \multicolumn{9}{|c|}{ Occupational UV exposure (high versus moderate exposure) } \\
\hline 44th $-<60$ th percentile (2.9-532.1 SED) & 40 & 63 & Ref. & Ref. & Ref. & Ref. & Ref. & Ref. \\
\hline$\geq 90$ th percentile ( $\geq 5870.5$ SED) & 26 & 26 & 1.87 & $0.94-3.73$ & 0.074 & 1.88 & $0.94-3.75$ & 0.072 \\
\hline
\end{tabular}

a number of cases and controls (contr)

b adjusted for age, age ${ }^{2}$, sex, phototype

c adjusted for age, age ${ }^{2}$, sex, phototype and non-occupational UV exposure 


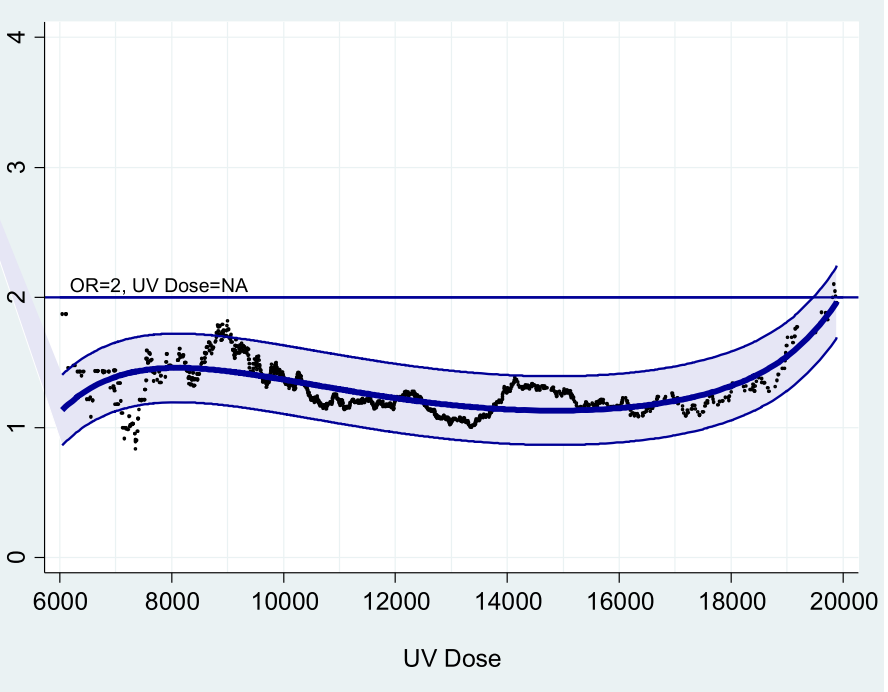

Fig. 1 Empirical odds ratios. Dose-response curves with 95\%-confidence band: dose-response relationship for total UV exposure. Odds ratios adjusted for sex, age, age ${ }^{2}$ and Fitzpatrick phototype using conditional logistic regression model (best fit): OR $=8.47 \mathrm{E}-9 *$ dose ${ }^{2}-125,261,133 * 1 /$ dose $^{2}-4.93 *$ LOG (dose) $+1.25 E-9 *$ EXP (dose) +47.13 (corrected $\left.R^{2}=0.481\right)$. NA = not applicable

commonly sun-exposed body sites. This is confirmed by reports in the literature, where not only poor tanners with Fitzpatrick phototype 1 and 2 but also good tanners with darker phototypes were shown to be at substantial risk to develop BCC when highly UV exposed [13, 22-25]. However, reported risk estimates varied from no risk increase up to more than 6-fold risk increase for BCC development in light phototypes compared to darker phototypes [22-
32]. These diverging results are probably due to different study designs, patient populations included (Northern/ South Europa, South America, Australia), latitude, determination of phototype (by measurements, physician- or patient-reported) and statistical methods used. Based on the evidence in the literature and on our data we recommend that prevention strategies must address all outdoor workers regardless of phototype.

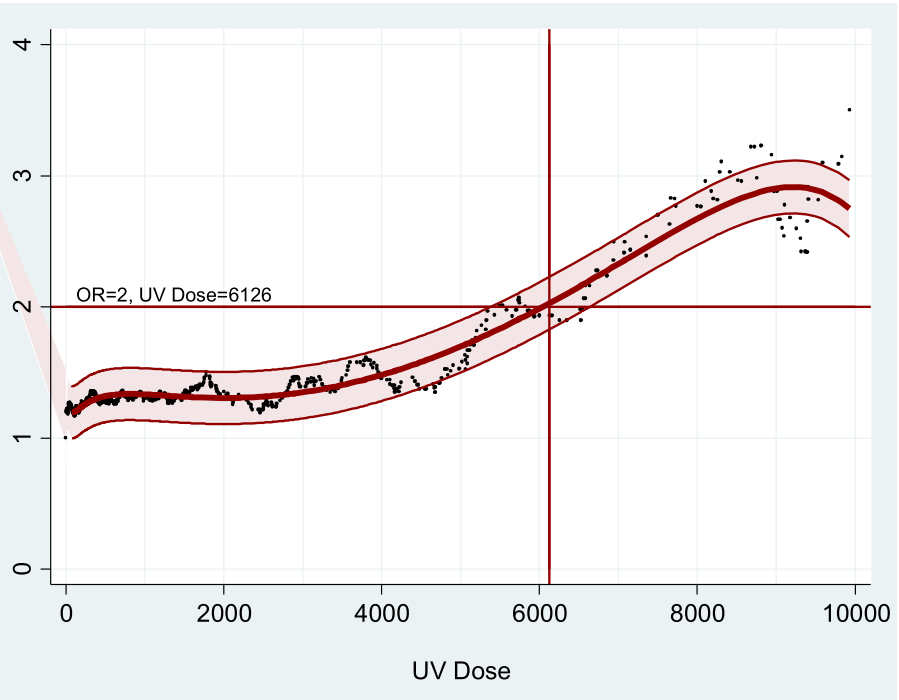

Fig. 2 Empirical odds ratios. Dose-response curves with 95\%-confidence band: dose-response relationship for occupational UV exposure. Legend: Odds ratios adjusted for sex, age, age squared, Fitzpatrick phototype and private UV exposure using conditional logistic regression model (best fit): $O R=-0.10 *$ SQRT (dose) $-58.76 * 1 /$ dose $+66.51 * 1 /$ dose $^{2}-37.35 * 1 /$ SQRT (dose) $+6.32 \mathrm{E}-6 * 1 /$ (dose * SQRT (dose) $)+1.81 *$ LOG (dose) $5.52 \mathrm{E}-5 * \operatorname{EXP}($ dose $)-9.28$ (corrected $\left.R^{2}=0.944\right)$ 


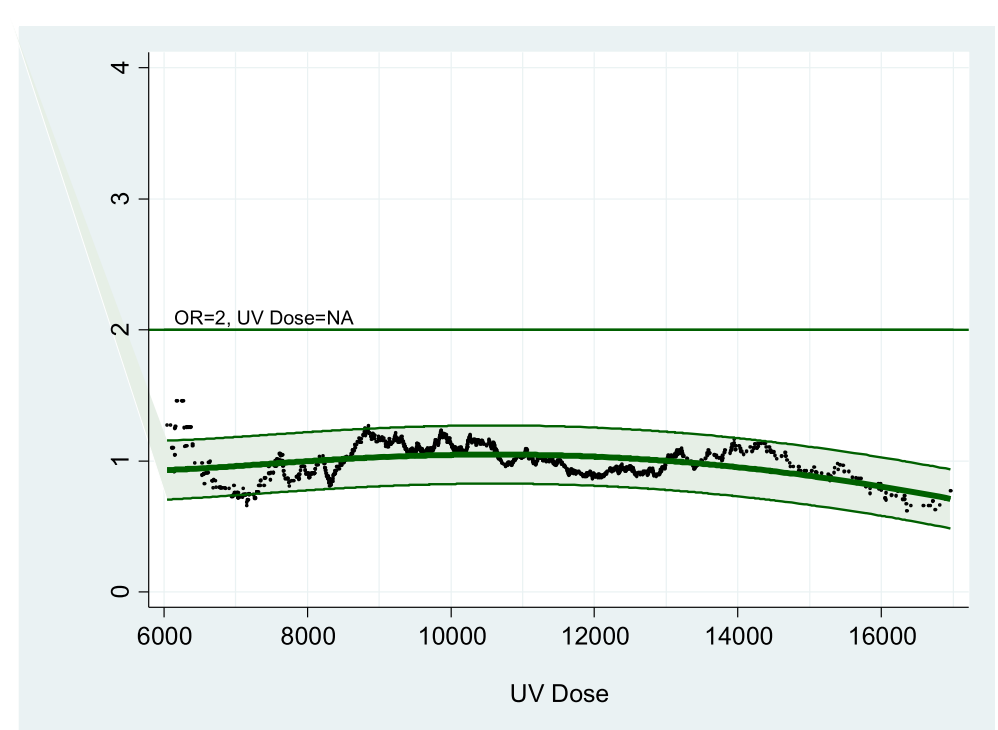

Fig. 3 Empirical odds ratios. Dose-response curves with 95\%-confidence band: dose-response relationship for private UV exposure. Legend: Odds ratios adjusted for sex, age, age squared and Fitzpatrick phototype using conditional logistic regression model (best fit): OR $=-5.73 \mathrm{E}-9 *$ dose ${ }^{2}+$ $20,282,607 * 1 /$ dose $^{2}+1.65 *$ LOG (dose) -13.78 (corrected $\left.R^{2}=0.199\right)$. NA = not applicable

\section{Occupations under risk}

Among BCC cases, we observed a significantly lower school education. A previous study found that outdoor work is associated with lower education [33]. This must be borne in mind when developing target group-specific prevention strategies.

\section{Dose-response relationship between occupational and non-occupational UV exposure and BCC risk}

In our study population, we quantified the association of cumulative UV exposure and BCC risk for occupational, non-occupational and total UV exposure. The doseresponse curve for occupational UV exposure, controlled for sex, age, age ${ }^{2}$, Fitzpatrick phototype, and nonoccupational UV exposure showed a positive doseresponse relationship up to approximately 8500 SED, which leveled off between 8500 and 9500 SED and fell afterwards. The risk doubled at a cumulative occupational UV dose of 6126 SED. This level was considerably lower compared to the recent analysis of the entire study population of 836 participants suffering from BCC at commonly sun-exposed and non-sun-exposed areas of the skin, where the risk to develop BCC doubled at 7945 SED [10]. The lower doubling dose in the present analysis is explained by the additional adjustment for sex, age, age ${ }^{2}$, Fitzpatrick phototype and non-occupational UV exposure and the restriction to $\mathrm{BCC}$ cases in commonly sun-exposed body sites. This result is consistent with the higher BCC risk estimates for the current analysis in 643 out of 836 cases.

The non-occupational UV dose-response curve showed a weaker rise and a broader plateau not reaching a risk- doubling dose. Our findings are in accordance with the literature, where comparable dose-response curves for BCC risk and total hours of sun exposure were identified in several studies [13, 34, 35]. It was speculated that subgroup effects introduced a complex dose-response relationship and thereby possibly a decreasing $\mathrm{BCC}$ risk at high doses [34]. Poor tanners (never tan or more often burn than tan) compared to good tanners exhibited different risk profiles. While poor tanners showed a plateau curve as mentioned above, good tanners seemed to have a steadily increasing risk to develop BCC [13].

In the present analysis of 643 cases with incident BCC in commonly sun-exposed body sites and their controls yielded a doubled risk of disease occurrence for occupational, but not non-occupational UV exposure. We assume that the decisive point leading to risk doubling by occupational UV exposure is the acquisition of high UV doses in shorter time intervals during occupational outdoor work (e.g. 6000 SED in 15 years of outdoor work, [400 SED/year]) compared to the cumulative UV exposure during entire lifetime (e.g. 9100 SED private exposure in a 70-year old person [130 SED/year]). This assumption is supported by recent evidence of high occupational UV exposure levels in various outdoor occupations by Wittlich et al. $[8,36]$

\section{Strengths and limitations}

The strength of our study is based on the fact that we analyzed a population-based sample of participants with incident histologically confirmed BCC recruited from a nationwide dermatologist network and matched controls [10]. By excluding patients with recurrent BCC, we 
reduced possible bias introduced by behavior and exposure change after the diagnosis of skin cancer. The population-based study design ensures a high degree of representativeness for the general population in Germany and Central Europe compared to previous hospital-based cohorts [9].

It is difficult to quantify the exact amount of sun exposure without taking UV dose measurements. To ensure data quality and completeness the computer-based, standardized, well-elaborated interviews were performed by trained interviewers. By this, we overcame limitations of self-administered questionnaires like bias due to missing data. Moreover, we ensured that all individual life periods were taken into account.

We cannot entirely exclude detection bias introduced by different observers, because interviews concerning occupational and non-occupational UV exposure were not performed by the same investigator at the sites. However, physicians assessing non-occupational UV exposure, as well as experts of the German Social Accident Insurance Institutions performing occupational UV exposure assessments were trained beforehand, thereby decreasing the risk of detection bias.

To control for confounders, cases and controls were matched. Moreover, the multivariate regression analysis was adjusted for additional confounders. Unlike previously published research $[9,37]$, we assessed occupational and non-occupational UV exposure in detail during all periods of life using precise and valid instruments recently developed for this purpose $[10,36,38]$. However, due to the retrospective collection of the UV exposure data, recall bias cannot be excluded entirely due to the difficulty to remember past-time sun behavior and exposure.

To reduce recall bias, the participants were asked in the invitation letter to consider the major stages of their private and occupational life prior to the interview at the clinic, as described in detail in the methods section. Moreover, occupational UV exposure was assessed more precisely compared to previous studies by using insurance documents on the duration of outdoor occupations.

However, selection bias might have played a role within the control group, which had been offered a free dermatological full body skin check as an incentive for study participation. This offer could have influenced participants with preexisting suspicious lesions and sun worshippers worried about possible cancer risk to seek medical advice. As a result, an underestimation of the association of BCC risk and occupational and nonoccupational UV exposure could have occurred. On the other hand, it cannot be completely excluded that the study especially attracted healthy controls, leading to an overestimation of the association of BCC risk and UV exposure ("healthy volunteer effect").
This study demonstrates an effect of cumulative occupational UV exposures on a qualitative and quantitative level. However, in particular the absolute UV exposure values could be questioned. The contribution of high intermittent, childhood and juvenile UV exposures may have been underestimated and, together with a possible differential recall bias of occupational and non-occupational UV exposures the dose response relationship of BCC risk and UV exposure could have been influenced.

Despite the remaining limitations, we believe that our study confirms our previous report indicating that the development of BCC in outdoor workers is dependent on high occupational solar UV exposure [10].

\section{Conclusion}

The risk to develop BCC due to high occupational UV exposure at capillitium, face, lip, neck, dorsum of the hands, forearms outside, and décolleté is doubled consistently, irrespective of phototype, tumor localization and histological subtype. Most commonly affected body sites were head and neck and the décolleté. There was a positive dose-response relationship of occupational UV exposure and BCC risk at capillitium, face, lip, neck, dorsum of the hands, forearms outside, décolleté. The $\mathrm{BCC}$ risk-doubling dose adjusted for age, age ${ }^{2}$, sex and non-occupational UV exposure was 6126 SED. Our study confirms our previous report that high occupational UV exposure in outdoor work is a relevant risk factor for BCC development.

\section{Supplementary information}

Supplementary information accompanies this paper at https://doi.org/10. 1186/s12995-020-00279-8.

Additional file 1: Table S1. Association of UV exposure and risk for BCC in cases with histopathological subtype nodular and matched controls ( $n=403$ in each group). Table S2. Association of UV exposure and risk for BCC in cases with histopathological subtype sclerodermiform and matched controls ( $n=145$ in each group). Table S3. Association of UV exposure and risk for BCC in cases with histopathological subtype superficial and matched controls ( $n=28$ in each group).

\section{Abbreviations \\ BCC : Basal cell carcinoma; SED : Standard erythema dose; OR : Odds ratio; CI : Confidence interval}

\section{Acknowledgements}

We would like to thank all individuals for participation in the study. We thank the clinical staff in the study centres in Dresden, Heidelberg, Erlangen-Nürnberg, Jena, Mainz, Bonn, Hornheide, Hamburg and Osnabrück for supporting FB 181 as well as the members of the included outpatient care network. Furthermore, we thank the professional staff of preventive services of the following employers' liability insurance associations for collaboration: Saxony Accident Insurance Fund, Federal Accident Insurance Fund, North Rhine-Westphalian Accident Insurance Fund, Social Insurance for Agriculture, Forestry and Gardening, Administrative Professional Association, Insurance Association of Wood and Metal Processing, Insurance Association of Energy, Textile, Electrics and Media Processing, Insurance Association of Construction Industry, Insurance Association of Natural Resources and Chemical Industry. We thank the entire scientific advisory board for methodological counselling. 
The FB 181 Study Group includes all authors and the following persons (alphabetically ordered):

Thomas Bieber, MD PhD (Department of Dermatology and Allergology, Christine Kühne Center for Allergy Research and Education, University of Bonn, Bonn, Germany); Sonja Bonness, MD (Institute of Interdisciplinary Dermatological Prevention and Rehabilitation (iDerm) at the University of Osnabrück, Employer's Liability Insurance Association Clinics Hamburg, Hamburg, Germany); Beate Brecht (Centre of Evidence-based Healthcare, University Hospital and Medical Faculty Carl Gustav Carus, TU Dresden, Germany); Stephan Grabbe, MD (Department of Dermatology, University Medical Centre, Johannes-Gutenberg University, Mainz, Germany); Denise Küster (Centre of Evidence-based Healthcare, University Hospital and Medical Faculty Carl Gustav Carus, TU Dresden, Germany), MPH; Linda Ruppert, MPH (Department of Clinical Social Medicine, Occupational and Environmental Dermatology, University of Heidelberg, Heidelberg, Germany); Victoria Stephan (Centre of Evidence-based Healthcare, University Hospital and Medical Faculty Carl Gustav Carus, TU Dresden, Germany); Anja Thielitz, MD (Institute of Interdisciplinary Dermatological Prevention and Rehabilitation (iDerm) at the University of Osnabrück, Employer's Liability Insurance Association Clinics Hamburg, Hamburg, Germany); Elisabeth Zimmermann (Department of Clinical Social Medicine, Occupational and Environmental Dermatology, University of Heidelberg, Heidelberg, Germany).

\section{Authors' contributions}

SJ, DTL, HE developed the concept of the study and the study design. HE, $\mathrm{HL}$ analysed the patient data. BA, HE, HL, SJ, SA, DTL, DH, LS, EP, JSM, FM, BT, $\mathrm{KP}, \mathrm{WM}, \mathrm{GW}$, WS with the input of all authors interpreted the patient data. $A B$ wrote the draft manuscript. SHJ, DBS, WW, GM, BK, BI, LB, BR, AJP performed the acquisition, interviews and clinical examination of cases and controls. All authors read and approved the final manuscript.

\section{Funding}

Sources of funding: The study was funded by the German Social Accident Insurance (DGUV, study ID FB-181). The funder had no active role in the design, conduct, analysis and interpretation of the study, or the writing of the study report. The publication of this manuscript was supported by Stiftung Hochschulmedizin Dresden (https://stiftung-hochschulmedizin.de). The study did not receive other funding or pharmaceutical industry support. Open access funding provided by Projekt DEAL.

\section{Availability of data and materials}

The datasets generated and/or analysed during the current study are available on request.

\section{Ethics approval and consent to participate}

Approval of the ethic committees of all participating sites was available before the first participant was included. All participants provided written informed consent prior to enrolment in the study.

\section{Consent for publication}

\section{Not applicable.}

\section{Competing interests}

Funds.

SJ received funding for educational lectures from the German Social Accident Insurance (DGUV). Outside the scope of this paper, he received institutional funding for investigator-initiated research from Pfizer, Novartis, ALK, and Sanofi.

BA received funding for educational lectures, workshops and expert opinions from the German Social Accident Insurance (DGUV). Outside the scope of this paper, she received grants for research paid to the institution, fees for lectures and advisory boards from Novartis, Leo, Sanofi, Biofrontera, Lofarma, Circassia, ALK, Incyte Corporation, Genentech, Shire, and Atopixs Therapeutics Ltd.

$E P, F M, B T, B R, L B, D G S, S H J$ received payments for expert witness for one of the collaborating as well as for other funds. BI, AP, KP, DH, LS, JSM, HL, HE do not declare any COI.

Employer.

FM and BT declare no conflicts of interest. As staff of the Institute for Prevention and Occupational Medicine (IPA), they are employed at the 'Berufsgenossenschaft Rohstoffe und chemische Industrie' (BG RCI), a public body which is a member of the study's main sponsor, the German Social Accident Insurance. IPA is an independent research institute of the RuhrUniversity Bochum. The authors are independent of the German Social Accident Insurance regarding study design, access to the collected data, responsibility for data analysis and interpretation, and the right to publish. The views expressed in this paper are those of the authors and not necessarily those of the sponsor.

The other authors declare no potential conflicts of interest.

\section{Author details}

${ }^{1}$ Department of Dermatology, University AllergyCentre, Medical Faculty Carl Gustav Carus, Technical University Dresden, Fetscherstr 74, D-01307 Dresden, Germany. ${ }^{2}$ Centre of Evidence-based Healthcare, University Hospital and Medical Faculty Carl Gustav Carus, Technical University Dresden, Dresden, Germany. ${ }^{3}$ Institute and Policlinic for Occupational and Social Medicine, Faculty of Medicine Carl Gustav Carus, Dresden, Germany. ${ }^{4}$ nstitute and Outpatient Clinics of Occupational and Social Medicine, Medical Faculty Carl Gustav Carus, Technical University Dresden, Dresden, Germany. ${ }^{5}$ Department of Dermatology, Dermatological Radiotherapy and Dermatohistopathology, Special Clinics Hornheide, Münster, Germany. ${ }^{6}$ Department of Dermatology, University Hospital Jena, Jena, Germany. ${ }^{7}$ Institute and Outpatient Clinic of Occupational, Social and Environmental Medicine,

Friedrich-Alexander-University Erlangen-Nürnberg, Erlangen, Germany. ${ }^{8}$ Institute of Occupational, Social and Environmental Medicine, University Medical Centre, Johannes-Gutenberg University Mainz, Mainz, Germany. ${ }^{9}$ Department of Dermatology, Environmental Medicine and Health Theory, University of Osnabrück and Institute of Interdisciplinary Dermatological Prevention and Rehabilitation (iDerm) at the University of Osnabrück, Osnabrück, Germany. ${ }^{10}$ Institute for Prevention and Occupational Medicine of the German Social Accident Insurance (DGUV), Institute of Ruhr University Bochum (IPA), Bochum, Germany. " ${ }^{1}$ Department of Clinical Social Medicine, Occupational and Environmental Dermatology, University of Heidelberg, Heidelberg, Germany. ${ }^{12}$ Department of Dermatology, University Medical Centre, Johannes-Gutenberg University Mainz, Mainz, Germany.

${ }^{13}$ Department of Dermatology and Allergology, Christine Kühne Center for Allergy Research and Education, University of Bonn, Bonn, Germany.

${ }^{14}$ Department of Radiation, Institute of Occupational Health and Safety of the German Social Accident Insurance (DGUV), Sankt Augustin, Germany.

${ }^{15}$ Department of Dermatology, Experimental Photobiology, Medical Faculty

Carl Gustav Carus, Technical University Dresden, Dresden, Germany.

Received: 29 November 2019 Accepted: 12 August 2020

Published online: 10 September 2020

\section{References}

1. Paterson SK, Godsmark CN. Heat-health vulnerability in temperate climates: lessons and response options from Ireland. Global Health. 2020;16:29.

2. Intranuovo G, Schiavulli N, Cavone D, Birtolo F, Cocco P, Vimercati L, Macinagrossa L, Giordano A, Perrone T, Ingravallo G, Mazza P, Strusi M, Spinosa C, Specchia G, Ferri GM. Assessment of DNA damages in lymphocytes of agricultural workers exposed to pesticides by comet assay in a cross-sectional study. Biomarkers. 2018;23:462-73.

3. Al-Bouwarthan M, Quinn MM, Kriebel D, Wegman DH. A field evaluation of construction workers' activity, hydration status, and heat strain in the extreme summer heat of Saudi Arabia. Ann Work Expo Health. 2020. https://doi.org/10.1093/annweh/wxaa029 [Epub ahead of print].

4. Vimercati L, Gatti MF, Baldassarre A, Nettis E, Favia N, Palma M, Martina GL, Di Leo E, Musti M. Occupational Exposure to Urban Air Pollution and Allergic Diseases. Int J Environ Res Public Health. 2015;12:12977-87.

5. Saladi RN, Persaud AN. The causes of skin cancer: a comprehensive review. Drugs Today. 2005:41:37-53.

6. Knuschke P. Forschungsergebnisse auf dem Gebiet der beruflichen solaren UV-Exposition. Dermatol Beruf Umwelt. 2011;59:78-83.

7. Thieden E, Philipsen PA, Heydenreich J, Wulf HC. UV radiation exposure related to age, sex, occupation, and sun behavior based on time-stamped personal dosimeter readings. Arch Dermatol. 2004;140:197-203.

8. Wittlich M. GENESIS-UV: neue Daten zur UV-Strahlenexposition an verschiedenen Arbeitsplätzen. Dermatol Beruf Umwelt. 2017;65:69-72.

9. Bauer A, Diepgen TL, Schmitt J. Is occupational solar ultraviolet irradiation a relevant risk factor for basal cell carcinoma? A systematic review and metaanalysis of the epidemiological literature. Br J Dermatol. 2011;165:612-25. 
10. Schmitt J, Haufe E, Trautmann F, et al. Occupational UV-Exposure is a major risk factor for basal cell carcinoma: results of the population-based casecontrol study FB-181. J Occup Environ Med. 2018;60:36-43.

11. Fitzpatrick TB. Soleil et peau. J Méd Esthétique. 1975;2:33-4

12. Pelucchi C, Di Landro A, Naldi L, et al. Risk factors for histological types and anatomic sites of cutaneous basal-cell carcinoma: an Italian case-control study. J Invest Dermatol. 2007;127:935-44.

13. Rosso S, Zanetti R, Martinez C, et al. The multicentre south European study 'Helios'. II: different sun exposure patterns in the aetiology of basal cell and squamous cell carcinomas of the skin. Br J Cancer. 1996;73:1447-54.

14. Royston P, Sauerbrei W. Multivariable Model-building. A pragmatic approach to regression analysis based on fractional polynomials for modelling continuous variables. Chichester: Wiley; 2008.

15. Heenan PJ, Elder DJ, Sobin LH. Histological typing of skin tumours. In: WHO international histological classification of tumours series. 2nd ed. Berlin, Heidelberg, New York, Tokyo: Springer-Verlag; 1996. p. 48-51.

16. Bastiaens MT, Hoefnagel JJ, Bruijn JA, et al. Differences in age, site distribution, and sex between nodular and superficial basal cell carcinomas indicate different types of tumors. J Invest Dermatol. 1998;110:880-4.

17. DeVries E, Louwman M, Bastiaens MT, et al. Rapid and continuous increases in incidence rates of basal cell carcinoma in the South East Netherlands since 1973. J Invest Dermatol. 2004;123:634-8.

18. McCormack CJ, Kelly JW, Dorevitch AP. Differences in age and body site distribution of the histological subtypes of basal cell carcinoma: a possible indicator of differing causes. Arch Dermatol. 1997:133:593-6.

19. Scrivener $Y$, Grosshans E, Cribier B. Variations of basal cell carcinomas according to gender, age, location and histopathological subtype. $\mathrm{Br} J$ Dermatol. 2002;147:41-7

20. Betti R, Inselvini E, Carducci M, Crosti C. Age and site prevalence of histologic subtypes of basal cell carcinomas. Int J Dermatol. 1995;34:174-6.

21. Raasch BA, Buettner PG, Garbe C. Basal cell carcinoma: histological classification and body-site distribution. Br J Dermatol. 2006;155:401-7.

22. Gallagher RP, Hill GB, Bajdik CD, et al. Sunlight exposure, pigmentary factors, and risk of nonmelanocytic skin cancer. I. Basal cell carcinoma. Arch Dermatol. 1995;131:157-63.

23. Maia M, Proença NG, de Moraes JC. Risk factors for basal cell carcinoma: a case-control study. Rev Saude Publica. 1995;29:27-37.

24. Kricker A, Armstrong BK, English DR, Heenan PJ. Pigmentary and cutaneous risk factors for non-melanocytic skin cancer: a case-control study. Int J Cancer. 1991;48:650-62.

25. Vitaliano PP, Urbach F. The relative importance of risk factors in nonmelanoma carcinoma. Arch Dermatol. 1980;116:454-6.

26. Walther $U$, Kron M, Sander S, et al. Risk and protective factors for sporadic basal cell carcinoma: results of a two-centre case-control study in southern Germany. Clinical actinic elastosis may be a protective factor. $\mathrm{Br} J$ Dermatol. 2004;151:170-8

27. Corona R, Dogliotti E, D’Errico M, et al. Risk factors for basal cell carcinoma in a Mediterranean population: role of recreational sun exposure early in life. Arch Dermatol. 2001;137:1162-8.

28. Naldi L, DiLandro A, D'Avanzo B, Parazzini F. Host-related and environmental risk factors for cutaneous basal cell carcinoma: evidence from an Italian case-control study. J Am Acad Dermatol. 2000;42:446-52.

29. Rosso S, Joris F, Zanetti R. Risk of basal and squamous cell carcinomas of the skin in Sion, Switzerland: a case-control study. Tumori. 1999;85:435-42.

30. Hogan DJ, To T, Gran L, et al. Risk factors for basal cell carcinoma. Int J Dermatol. 1989;28:591-4

31. Gafà L, Filippazzo MG, Tumino R, et al. Risk factors of nonmelanoma skin cancer in Ragusa, Sicily: a case-control study. Cancer Causes Control. 1991;2: 395-9.

32. Kricker A, Armstrong BK, English DR, Heenan PJ. Does intermittent sun exposure cause basal cell carcinoma? A case-control study in Western Australia. Int J Cancer. 1995;60:489-94.

33. Trakatelli M, Barkitzi K, Apap C, Majewski S, De Vries E, EPIDERM group. Skin cancer risk in outdoor workers: a European multicenter case-control study. J Eur Acad Dermatol Venereol. 2016;30(Suppl 3):5-11.

34. Kricker A, Armstrong BK, English DR, Heenan PJ. A dose-response curve for sun exposure and basal cell carcinoma. Int J Cancer. 1995;60:482-8.

35. Strickland PT, Vitasa BC, West SK, et al. Quantitative carcinogenesis in man: solar ultraviolet B dose dependence of skin cancer in Maryland watermen. J Natl Cancer Inst. 1989;81:1910-3.
36. Wittlich M, Westerhausen S, Kleinespel P, Rifer G, Stoppelmann W. An approximation of occupational lifetime UVR exposure: algorithm for retrospective assessment and current measurements. J Eur Acad Dermatol Venereol. 2016;30(suppl 3):27-33.

37. Lindelöf B, Lapins J, Dal H. Shift in Occupational Risk for Basal Cell Carcinoma from Outdoor to Indoor Workers: A Large Population-based Case-control Register Study from Sweden. Acta Derm Venereol. 2017;97: 830-3.

38. Elsner P, Bauer A, Blome O, et al. Malignant skin tumors induced by UV irradiation: development and evaluation of legally relevant criteria for differentiating occupational and non-occupational causes. DGUV Research Project FB 170. Dermatol Beruf Umwelt. 2014;62:107-32.

\section{Publisher's Note}

Springer Nature remains neutral with regard to jurisdictional claims in published maps and institutional affiliations.
Ready to submit your research? Choose BMC and benefit from:

- fast, convenient online submission

- thorough peer review by experienced researchers in your field

- rapid publication on acceptance

- support for research data, including large and complex data types

- gold Open Access which fosters wider collaboration and increased citations

- maximum visibility for your research: over $100 \mathrm{M}$ website views per year

At BMC, research is always in progress.

Learn more biomedcentral.com/submissions 